\title{
SIMPLE CLOSED GEODESICS ON PINCHED SPHERES
}

\author{
WILHELM KLINGENBERG
}

Let $M$ be a compact simply connected $n$-dimensional riemannian manifold. If the values of the sectional curvature $K$ of $M$ satisfy the condition $\min K: \max K>1 / 4$ then $M$ is homeomorphic to the $n$-sphere $S^{n}$. We therefore call such a manifold a pinched sphere. Cf. [1], [3] for the proof of this so-called sphere theorem, and [2] for a complete exposition.

By multiplying the riemannian metric of such a manifold $M$ with an appropriate positive constant we obtain a manifold for which the relation

$$
1 / 4<\pi \leq K \leq 1
$$

holds. That is to say, such a manifold is curved stronger than the sphere $S^{n}(4)$ of constant curvature $1 / 4$ and curved less strongly than the unit sphere $S^{n}=S^{n}(1)$. This raises the problem of existence, on such a manifold, of simple closed geodesics of length between $2 \pi$ and $4 \pi$, the length of the great circles on $S^{n}(1)$ and $S^{n}(4)$ respectively. The example of the $n$-dimensional ellipsoid with pairwise different axes suggests that there may be, but not more than, $n(n+1) / 2$ such closed geodesics on a manifold $M$ satisfying (*).

In the present paper we shall first prove the following

Theorem 1. On a compact simply connected $n$-dimensional riemannian manifold $M$ satisfying (*) there exist at least $n$ simple closed geodesics with length in $[2 \pi, 2 \pi / \sqrt{\kappa}]$.

To formulate our next result, for each integer $n \geq 2$ we define the integer $g(n)$ as $2 n-s(n)-1$ with $s(n)=n-2^{h}, 0 \leq s(n)<2^{n}$. Note that for $n=2^{2 h}-1$ we have $s(n)=(n-1) / 2$ and hence $g(n) \geq(3 n-1) / 2$.

Theorem 2. Let $M$ be a compact simply connected $n$-dimensional riemannian manifold satisfying $(*)$ with $\kappa(\sim 0.64)$ as the solution of $2 \kappa \sin \frac{\pi}{2 \sqrt{\kappa}}$ $=1$. Then on $M$ there exist $g(n)$ simple closed geodesics of length in $[2 \pi, 4 \pi[$. If $\kappa(\sim 0.46)$ is the solution of $2 \sqrt{\kappa} \sin \frac{\pi}{2 \sqrt{\kappa}}=1$, then there exist at least $g(n-1)$ such geodesics on $M$.

Suppose that on $M$ the closed geodesics of length $<4 \pi$ are not isolated or are non-degenerate. If $(*)$ holds with $\kappa \sim 0.64$, then on $M$ there exist exactly

Communicated April 15, 1968. This paper was completed with a support from NSF grant GP-6974. 
$n(n+1) / 2$ simple closed geodesics of length in $[2 \pi, 4 \pi[;$ if $(*)$ holds with $\kappa \sim 0.46$, then there are at least $(n-1) n / 2$ such geodesics on $M$.

Remark. We certainly have not obtained the best lower bounds for $\kappa$ in our theorem. In particular, using more delicate arguments, it is possible to show the existence of $g(n)$ simple closed geodesics on a manifold $M$ satisfying (*) with $\kappa \sim 0.46$.

However, we want to stress that Theorem 2 does establish, for the first time, the existence of several simple or prime (i.e., not covered) closed geodesics on a manifold $M$ which is homeomorphic to the sphere $S^{n}(n>2)$ satisfying certain metric restrictions. In particular, the results of Morse (1934), Lyusternik (1947) and Alber (1957) (cf. [4] for precise references) do not imply the existence of more than one prime closed geodesic on such a manifold $M$, even under the hypothesis that there exists a homeomorphism $h: S^{n} \rightarrow M$ satisfying the so-called Morse condition. The reason for this is that these authors do not exclude the possibility that all the closed geodesics constructed in their papers are merely different coverings of the same underlying prime closed geodesic.

Only in the case that $M$ is homeomorphic to $S^{2}$, Lusternik and Schnirelmann showed in 1929 the existence of 3 simple closed geodesics, but did not discuss, however, the length of these geodesics in the case that the gaussian curvature on $M$ satisfies a restriction of the type (*). It should also be mentioned that Fet in 1965 showed the existence of at least 2 prime geodesics on any compact riemannian manifold provided all the closed geodesics on $M$ are non-degenerate. Using quite delicate geometric arguments, the present author was recently able to show the existence of $g(n)$ simple closed geodesics on a manifold $M$ homemorphic to $S^{n}$ without making any assumptions on the metric or the curvature of $M$.

Going back to the theorems of the present paper, we want to give an outline of the methods employed in their proofs. In the first place, we construct closed geodesics on a compact riemannian manifold $M$ by the classical methods of using the homology of the space $\Pi(M)$ of unparametrized closed curves on $M$ modulo the subspace $\Pi^{\circ}(M)$ of the trivial closed curves (=point curves). We refer to our paper [4] for a modern and detailed version of this theory.

Since in our case $M$ is homemorphic to the sphere $S^{n}$, we know moreover that there exists a map $h_{\Gamma}:\left(\Gamma\left(S^{n}\right), \Gamma^{0}\left(S^{n}\right)\right) \rightarrow\left(\Pi(M), \Pi^{0}(M)\right)$ of the space $\Gamma\left(S^{n}\right)$ of the circles on $S^{n}$, which is considered as a subspace of $\Pi\left(S^{n}\right)$ modulo the subspace $\Gamma^{0}\left(S^{n}\right)$ of the point circles. The important fact is that this map is injective in $Z_{2}$-homology and therefore gives us $n(n+1) / 2$ $=\operatorname{dim} H^{*}\left(\Gamma\left(S^{n}\right), \Gamma^{0}\left(S^{n}\right)\right)$ non-homologous cycles in $\Gamma(M) \bmod \Gamma^{0}(M)$.

Secondly, we use a great deal of information about simply connected compact riemannian manifolds $M$ satisfying (*), which leads to the proof of the sphere theorem. In particular, we use in an essential manner the fact that on 
such a manifold $M$ every non-trivial geodesic loop has length at least $2 \pi$.

By linking together the above information we construct a homeomorphism $h: S^{n} \rightarrow M$ with $M$ satisfying the hypothesis of the theorems, which carries circles into curves of length $<4 \pi$.

1. We recollect the basic facts on the construction of closed geodesics on a compact riemannian manifold $M$ homemorphic to $S^{n}$. For a detailed exposition we refer to [4].

First of all, for any compact riemannian manifold $M$ we have the space $\Lambda(M)$ of parametrized closed curves. $\Lambda(M)$ is a Hilbert manifold, and the riemannian metric on $M$ induces on $\Lambda(M)$ a riemannian metric and a differentiable function, the energy integral $E$. The orthogonal group $O(2)$ operates continuously on $\Lambda(M)$, the orbit of an element $f=(f(t)), t \in S^{1}=[0,1] /$ $\{0,1\}$, being formed by the curves $(f( \pm t+c)), c \in S^{1}, t \in S^{1}$. Let $\pi: \Lambda(M)$ $\rightarrow \Pi(M)=\Lambda(M) / O(2)$ be the quotient map, where $\Pi(M)$ is called the space of unparametrized closed curves on $M$. Since the $O(2)$-action on $\Lambda(M)$ is compatible with the riemannian metric and the function $E$ on $\Lambda(M)$, these objects are also defined on $\Pi(M)$ although $\Pi(M)$ is not a manifold.

For $c \geq 0$, we denote by $\Pi^{c-}(M)$ the set of $g \in \Pi(M)$ with $E(g)<c$.

Consider now, in particular, the sphere $S^{n}$ of constant curvature. Then $\Pi\left(S^{n}\right)$ contains the subspace $\Gamma\left(S^{n}\right)$ of the circles on $S^{n}$ with $\Gamma^{0}\left(S^{n}\right)=\Gamma\left(S^{n}\right)$ $\cap \Pi^{0}\left(S^{n}\right)$, the subspace of point circles, isomorphic to $S^{n}$. An important fact is that the inclusion $\left(\Gamma\left(S^{n}\right), \Gamma^{0}\left(S^{n}\right)\right) \rightarrow\left(\Pi\left(S^{n}\right), \Pi^{0}\left(S^{n}\right)\right)$ induces an injective homomorphism in $Z_{2}$-homology, carrying subordinated homology classes into themselves. Here, if $z^{\prime}$ and $z$ are non-trivial homology classes of $\Pi(M) \bmod$ $\Pi^{\circ}(M)$ and there exists a cohomology class $\phi$ in $\Pi(M)-\Pi^{\circ}(M)$ with $z^{\prime}=z \cap \phi$ then $z^{\prime}$ is said to be subordinated to $z$. Here we make use of the pairing by the cap product of $H_{*}\left(\Pi(M), \Pi^{\circ}(M)\right) \times H^{*}\left(\Pi(M)-\Pi^{\circ}(M)\right)$ into $H_{*}\left(\Pi(M), \Pi^{\circ}(M)\right)$ (cf. [4]).

$H_{*}\left(\Gamma\left(S^{n}\right), \Gamma^{0}\left(S^{n}\right)\right)$ has dimension $n(n+1) / 2$, and $g(n)$ of the homology classes are pairwise subordinated.

Let now $M$ be homeomorphic to $S^{n}$. Then there exists a homeomorphism $h: S^{n} \rightarrow M$ such that $f \in \Lambda\left(S^{n}\right)$ implies $h \circ f \in \Lambda(M)$, and the map $h_{A}: \Lambda\left(S^{n}\right)$ $\rightarrow \Lambda(M)$ so defined is differentiable. Let $h_{\Pi}: \Pi\left(S^{n}\right) \rightarrow \Pi(M)$ be the induced map on the quotient space. Then $h_{\Gamma}=h_{n} \mid \Gamma\left(S^{n}\right):\left(\Gamma\left(S^{n}\right), \Gamma^{0}\left(S^{n}\right)\right) \rightarrow(\Pi(M)$, $\left.\Pi^{\circ}(M)\right)$ carries $n(n+1) / 2$ pairwise non-homologous cycles in the space of circles into such cycles of $\Pi(M) \bmod \Pi^{\circ}(M)$, among which $g(n)$ are pairwise subordinated.

If $c^{*}>0$ such that $h_{r}\left(\Gamma\left(S^{n}\right)\right) \subset \Pi^{c^{*}-}(M)$, then the critical number $c(z)$ of each of the homology classes $z$ represented by one of the cycles coming from the space of the circles on $S^{n}$ is less than $c^{*}$, i.e., there exists a closed geodesic of length $\sqrt{2 c(z)}<\sqrt{2 c^{*}}$.

2. Consider a compact riemannian manifold $M$ satisfying (*). Then the following holds (cf. [2]): 
The maximal distance $d\left(q_{0}, q_{1}\right)$ between two points of $M$ is $\leq \pi / \bar{\kappa}<2 \pi$; if the upper bound of $d\left(q_{0}, q_{1}\right)$ is attained then $M$ is isometric to the sphere $S(\kappa)$ of constant curvature $\kappa$. Moreover, for every point $q \in M$, the restriction of the exponential map $\exp _{q}: M_{q} \rightarrow M$ to the open disc $D_{\pi}(q)$ of radius $\pi$ around the origin $q$ of $M_{q}$ is an injective differentiable map. This is equivalent essentially to the fact that every non-trivial geodesic loop on $M$ has length $\geq 2 \pi$.

Let now $q_{0}, q_{1}$ be two points on $M$ with maximal distance. Then define on $M$ the subsets (which may be called half- $M$ 's with respect to $\left(q_{0}, q_{1}\right)$ )

$$
\begin{aligned}
& H M\left(q_{0}\right)=\left\{q \in M \mid d\left(q, q_{0}\right) \leq d\left(q, q_{1}\right)\right), \\
& H M\left(q_{1}\right)=\left\{q \in M \mid d\left(q, q_{1}\right) \leq d\left(q, q_{0}\right)\right),
\end{aligned}
$$

and call $\operatorname{EM}\left(q_{0}, q_{1}\right)=H M\left(q_{0}\right) \cap H M\left(q_{1}\right)$ the equator of $M$ with respect to $\left(q_{0}, q_{1}\right)$.

$H M\left(q_{0}\right)$ and $H M\left(q_{1}\right)$ belong to $\exp _{q_{0}}\left(D_{z}\left(q_{0}\right)\right)$ and $\exp _{q_{1}}\left(D_{z}\left(q_{1}\right)\right)$, respectively. The counter images in $M_{q_{0}}$ and $M_{q_{1}}$, respectively,

$$
\bar{\Delta}^{n}\left(q_{0}\right)=\left(\exp _{q_{0}} \mid D_{n}\left(q_{0}\right)\right)^{-1} H M\left(q_{0}\right), \quad \bar{\Delta}^{n}\left(q_{1}\right)=\left(\exp _{q_{1}} \mid D_{x}\left(q_{1}\right)\right)^{-1} H M\left(q_{1}\right)
$$

have the property that every ray, starting from the origin $q_{0}$ (resp. $q_{1}$ ), meets $\Sigma^{n-1}\left(q_{0}\right)=\partial \bar{\Delta}^{n}\left(q_{0}\right)$ (resp. $\left.\sum^{n-1}=\partial \bar{\Delta}^{n}\left(q_{1}\right)\right)$ at precisely one point.

The $\Sigma^{n-1}\left(q_{0}\right)$ and $\Sigma^{n-1}\left(q_{1}\right)$ are differentiably embedded $(n-1)$-spheres in $M_{q_{0}}$ and $M_{q_{1}}$, respectively. We now define the diffeomorphism

$$
\partial \tilde{\sigma}_{0}: S_{\pi / 2}^{n-1}\left(q_{0}\right) \rightarrow \Sigma^{n-1}\left(q_{0}\right)
$$

of the sphere of radius $\pi / 2$ and center $q_{0}$ in $M_{q_{0}}$ onto $\Sigma^{n-1}\left(q_{0}\right)$ by associating with a point on this sphere the point at which the ray through the former point meets $\sum^{n-1}\left(q_{0}\right)$. By extending $\partial \tilde{\sigma}_{0}$ linearly to the interior $D_{z / 2}^{n}\left(q_{0}\right)$ of $S_{\pi / 2}^{n-1}\left(q_{0}\right)$ we obtain the diffeomorphism

$$
\tilde{\sigma}_{0}: \bar{D}_{\pi / 2}^{n}\left(q_{0}\right) \rightarrow \bar{\Delta}^{n}\left(q_{0}\right) .
$$

We now use these constructions to define a homeomorphism $h: S^{n} \rightarrow M$. First of all, we choose in $S^{n}$ a pair $\left(p_{0}, p_{1}\right)$ of diametral points, and denote by $H S^{n}\left(p_{0}\right)$ and $H S^{n}\left(p_{1}\right)$ the halfspheres with centers $p_{0}$ and $p_{1}$, respectively. $E S^{n}\left(p_{0}, p_{1}\right)=H S^{n}\left(p_{0}\right) \cap H S^{n}\left(p_{1}\right)$ is the equator of $S^{n}$ with respect to $\left(p_{0}, p_{1}\right)$. Moreover, we fix an isometry $i=i_{p_{0}}: S_{p_{0}}^{n} \rightarrow M_{q_{0}}$ of the tangent space of $S^{n}$ at $p_{0}$ onto the tangent space of $M$ at $q_{0}$. We then define

$$
\sigma_{0}=\tilde{\sigma}_{0} \circ i: \bar{D}_{\bar{n} / 2}^{n}\left(p_{0}\right) \rightarrow \bar{\Delta}^{n}\left(q_{0}\right),
$$

and the diffeomorphism 


$$
h_{0}=\exp _{q_{0}} \circ \sigma_{0} \circ\left(\exp _{p_{0}} \mid D_{\pi}^{n}\left(p_{0}\right)\right)^{-1}: H S^{n}\left(p_{0}\right) \rightarrow H M\left(q_{0}\right)
$$

Since the equator $\operatorname{EM}\left(q_{0}, q_{1}\right)$ also belongs to $\exp _{q_{1}}\left(D_{\pi}^{n}\left(q_{1}\right)\right)$, we can define

$$
\partial \sigma_{1}=\left(\exp _{q_{1}} \mid D_{n}\left(q_{1}\right)\right)^{-1} \circ h_{0} \circ \exp _{p_{1}}: S_{n / 2}^{n-1}\left(p_{1}\right) \subset S_{p_{1}}^{n} \rightarrow \Sigma^{n-1}\left(q_{1}\right) \subset M_{q_{1}},
$$

which is a diffeomorphism. Let

$$
\sigma_{1}: \bar{D}_{\pi / 2}^{n}\left(p_{1}\right) \rightarrow \bar{\Delta}^{n}\left(q_{1}\right)
$$

be the linear extension of $\partial \sigma_{1}$. Note that $\sigma_{1}$ is a homeomorphism, but in general it will be a diffeomorphism only on $D_{x / 2}^{n}\left(p_{1}\right)-p_{1}$. We then define

$$
h_{1}=\exp _{q_{1}} \circ \sigma_{1} \circ\left(\exp _{p_{1}} \mid D_{n}^{n}\left(p_{1}\right)\right)^{-1}: H S^{n}\left(p_{1}\right) \rightarrow H M\left(q_{1}\right)
$$

Since $h_{0}\left|\partial H S^{n}\left(p_{0}\right)=h_{1}\right| \partial H S^{n}\left(p_{1}\right), h_{0}$ and $h_{1}$ together define the desired homeomorphism $h: S^{n} \rightarrow M$, which is differentiable outside of $\operatorname{ES}^{n}\left(p_{0}, p_{1}\right) \cup\left\{p_{1}\right\}$. The image of a half great circle from $p_{0}$ to $p_{1}$ will consist of a once-broken geodesic of length $\leq \pi / \sqrt{\kappa}$ with the corner in the middle on $\operatorname{EM}\left(q_{0}, q_{1}\right)$. It is possible to modify $h$ in such a manner that these corners are being smoothed out; however, in general it may not be possible to make $h$ smooth also at the point $p_{1}$. Note that $h$ induces a map from $S_{p_{1}}^{n}$ onto $M_{q_{1}}$, which is linear and bijective on each ray starting at $p_{1}$ but not necessarily linear on all of $S_{p_{1}}^{n}$, and that there will be an obstruction to smooth out this map whenever $M$ is not diffeomorphic to $S^{n}$.

3. We can now prove Theorem 1. First of all, we recall from [4] that the space $\Gamma\left(S^{n}\right)-\Gamma^{0}\left(S^{n}\right)$ of non-trivial circles on $S^{n}$ can be viewed as the total space of a bundle $\gamma$ of open $(n-1)$-discs over the space $\Delta\left(S^{n}\right)$ of great circles on $S^{n}$ by mapping each circle onto its parallel great circle. $\Delta\left(S^{n}\right)$ is isomorphic to the Grassmann manifold $G(2, n-1)$ of 2-planes in $R^{n+1}$ and $\gamma$ is the disc bundle of the canonical $(n-1)$-bundle $\gamma^{n-1}$ over $G(2, n-1)$. With $\Gamma^{0}\left(S^{n}\right)$ denoting the subspace of $\Gamma\left(S^{n}\right)$ formed by the trivial (or point) circles it follows that $\Gamma\left(S^{n}\right) \bmod \Gamma^{0}\left(S^{n}\right)$ can be interpreted as the Thom space of $\gamma^{n-1}$.

Let now $p_{0}, p_{1}$ be a pair of diametral points on $S^{n}$ as in $\S 2$. The set of great circles on $S^{n}$, which pass through $p_{0}$ and $p_{1}$, forms a $(n-1)$-cycle $x_{n-1}$ of $\Delta\left(S^{n}\right)$, and $x_{n-1}$ is dual to the $(n-1)$-th power of the 1-dimensional generator of the cohomology ring of $\Delta\left(S^{n}\right)=G(2, n-1)$. Therefore, the subset $y_{2 n-2}$ of $\Gamma\left(S^{n}\right)$ formed by the circles parallel to a great circle belonging to $x_{n-1}$ is modulo the subspace of point circles a $(2 n-2)$-cycle $y_{2 n-2}$ which contains $(n-1)$ cycles $y_{n-1}, y_{n}, \cdots, y_{2 n-3}$ such that the $n$ cycles $y_{i}, n-1 \leq i$ $\leq 2 n-2$, are pairwise subordinated.

Consider now $\Gamma\left(S^{n}\right)$ as subset of $\Pi\left(S^{n}\right)$. Then the $y_{i}$ form $n$ pairwise subordinated cycles of $\Gamma\left(S^{n}\right) \bmod \Gamma^{0}\left(S^{n}\right)$. We replace the $y_{i}$ by homologous cycles $\tilde{y}_{i}$ as follows: Each non-trivial circle of $y_{2 n-2}$ meets the equator 
$\operatorname{ES}^{n}\left(p_{0}, p_{1}\right)$ at exactly two points. Replace the circle by the closed curve formed by the two half great circles going from $p_{0}$ to $p_{1}$ and passing through these two points. Replace a point circle of $y_{2 n-2}$, which necessarily belongs to $E S^{n}\left(p_{0}, p_{1}\right)$, by the closed curve formed by the half great circle from $p_{0}$ to $p_{1}$, which passes through the point curve and goes back to $p_{0}$ in the same way. Finally, join this curve to the point circle by a 1-parameter family of closed curves which consist of arcs of this half great circle, run through back and forth, and shrink towards the point circle.

If we replace, for each $i$, the circles in $y_{i}$ by the closed curves just described, we obtain the cycles $\tilde{y}_{i}$, homologous to $y_{i}$. Consider now the map $h: S^{n} \rightarrow M$ of $\S 2$. Then the induced map $h_{n}$ carries each closed curve of $\tilde{y}_{2 n-2}$ into a closed curve of length $\leq 2 \pi / \sqrt{\kappa}$, and we can introduce simultaneously on the elements of $h_{\Pi} \circ y_{2 n-2}$ a parameter proportional to the arc length by a homotopy in $\Pi(M)$. Hence, we can assume that $h_{\Pi} \circ y_{2 n-2}$ consists entirely of curves having $E$-value $\leq 2 \pi^{2} / \kappa<8 \pi^{2}$.

From $\S 1$ now follows the existence of $n$ non-trivial closed geodesics of $E$ value $\leq 2 \pi^{2} / \kappa$, i.e., of length $\leq 2 \pi / \sqrt{\kappa}<4 \pi$. However such a geodesic is necessarily simple since every non-trivial geodesic loop on $M$ has length $\geq 2 \pi$.

4. For the proof of Theorem 2 we need an upper bound for the stretching of the length of a circle $b$ of $S^{n}$ under the map $h: S^{n} \rightarrow M$ constructed in $\S 2$. For this purpose we prove the

Proposition. Let $v$ be a tangent vector at a point $p^{\prime} \in H S^{n}\left(p_{0}\right)$ or tangent to one of the geodesic arcs of length $\pi / 2$ from $p_{1}$ to the equator $\operatorname{ES}^{n}\left(p_{0}, p_{1}\right)$. Then for the length $\left|h_{*} v\right|$ we have the relation

$$
\left|h_{*} v\right| \leq|v| / \sqrt{\kappa} \text {. }
$$

If $v$ is a tangent vector at a point $p^{\prime} \in H S^{n}\left(p_{1}\right)-\left\{p_{1}\right\}$ then

$$
\begin{aligned}
\left|h_{*} v\right| & \leq|v| \sin (r / \sqrt{\kappa}) /\left(\sqrt{\kappa} \sin r \sin \frac{\pi}{2 \sqrt{\kappa}}\right) \\
& \leq|v| /\left(\kappa \sin \frac{\pi}{2 \sqrt{\kappa}}\right),
\end{aligned}
$$

where $r=d_{M}\left(p^{\prime}, p_{1}\right)$.

For the proof of this Proposition we cover $S^{n}$ with two normal coordinate systems based at $p_{0}$ and $p_{1}$, respectively. The line element of $S^{n}$ is then given by $d s^{2}=d r^{2}+\sin ^{2} r d \phi^{2}$, where $d \phi^{2}$ is the line element of the unit sphere in either $S_{p_{0}}^{n}$ or $S_{p_{1}}^{n}$.

Since the geodesic segments of length $\pi / 2$ from $p_{0}$ to a point $p \in E S^{n}\left(p_{0}, p_{1}\right)$ are stretched by at most $1 / \sqrt{\kappa}$ we have in this case $h_{*} d r \leq d r / \sqrt{\kappa}$.

Consider now the geodesic segment $\sigma_{0}: 0 \leq r \leq \pi / 2, \phi=\phi_{0}$, from $p_{0}$ to a point $p \in E S^{n}\left(p_{0}, p_{1}\right)$. Then $\sin r d \phi$ can be considered as a normalized Jacobi 
field $\left(Y_{0}(r)\right), 0 \leq r \leq \pi / 2$, along $\sigma_{0}$, i.e., $Y_{0}(\mathrm{O})=0$ and $D Y_{0}(\mathrm{O}) / d r=1$. The image $h_{*} \sin r d \phi$ then will be a normalized Jacobi field $(Y(r)), 0 \leq \pi /\left(2 \sqrt{\kappa_{0}}\right)$ $\leq \pi /(2 \sqrt{\kappa})$ along the image segment $\sigma=h \sigma_{0}$ of length $\pi /\left(2 \sqrt{\kappa_{0}}\right)$. Since the sectional curvature $K$ along $\sigma$ is bounded from below by $\kappa$, the comparison theorem of Rauch [5] (cf. [2] for a complete proof) gives $|Y(r)| \leq\left|Y_{1}(r)\right|$, $0 \leq r \leq \pi /\left(2 \sqrt{\kappa_{0}}\right)$, where $\left(Y_{1}(r)\right)$ is the normalized Jacobi field $(1 / \sqrt{\kappa})$ $\sin (r \sqrt{\kappa})$ in the sphere $S^{n}(\kappa)$. That is to say, we have

$$
\left|h_{*} \sin r d \phi\right| \leq(1 / \sqrt{\kappa})\left|\sin \left(r \sqrt{\kappa} / \sqrt{\kappa_{0}}\right) d \phi\right| \leq(1 / \sqrt{\kappa})|\sin r d \phi|,
$$

which is ( $\mathrm{i}$ ).

It remains to consider the image $h_{*} \sin r d \phi$ of a normalized Jacobi field $\sin r d \phi$ along the segment $\sigma_{1}: 0 \leq r \leq \pi / 2, \phi=\phi_{1}$, of length $\pi / 2$ from $p_{1}$ to a point $p \in E S^{n}\left(p_{0}, p_{1}\right)$. Let $\pi /\left(2 \sqrt{\kappa_{0}}\right)$ be the length of the image segment $\sigma=h \sigma_{1}$, with $\pi / 2 \leq \pi /\left(2 \sqrt{\kappa_{0}}\right) \leq \pi /(2 \sqrt{\kappa})<\pi$. $h \sin r d \phi$ is again a Jacobi field $\left(Y\left(r / \sqrt{\kappa_{0}}\right)\right), 0 \leq r \leq \pi / 2$, along $\sigma$, but it is not necessarily normalized. However, we know that $\left|Y\left(\frac{\pi}{2 \sqrt{\kappa_{0}}}\right)\right| \leq 1 / \sqrt{\kappa}$. We consider now the Jacobi field

$$
\left(Y_{0}\left(r / \sqrt{\kappa_{0}}\right)\right)=\left(\sin (r / \sqrt{\kappa}) d \phi /\left(\sqrt{\kappa} \sin \frac{\pi}{2 \sqrt{\kappa}}\right)\right), \quad 0 \leq r \leq \pi / 2
$$

on $S^{n}$ (1) along a geodesic segment of length $\pi / 2 \sqrt{\pi}<\pi$. From the proof of Rauch's comparison theorem in [2] one knows that, since the sectional curvature $K$ along $\sigma$ satisfies $K \leq 1$, the function $\rho(r)=|Y(r / \sqrt{\kappa})| /\left|Y_{0}(r / \sqrt{\kappa})\right|$ is monotonely increasing with $r$ in the interval $[0, \pi / 2]$. Since $\rho(\pi / 2) \leq 1$ it follows that

$$
\begin{aligned}
\left|h_{*} v\right| & =|Y(r / \sqrt{\kappa})| \leq\left|Y_{0}(r / \sqrt{\kappa})\right| \\
& =\left[\sin (r / \sqrt{\kappa}) /\left(\sqrt{\kappa} \sin r \sin \frac{\pi}{2 \sqrt{\kappa}}\right)\right]|\sin r d \phi|,
\end{aligned}
$$

which is (ii).

5. We can now prove Theorem 2 . There is a well determined solution $\kappa \sim 0.64$ of $2 \kappa \sin \frac{\pi}{2 \sqrt{\kappa}}=1$. We fix $\kappa$ in this manner and consider the map $h: S^{n} \rightarrow M$ constructed in $\S 2$. From $\S 4$ it now follows that each circle of $S^{n}$ is carried under $h$ into a curve having length $\leq 4 \pi$; actually the length of the image of a circle is $<4 \pi$. This is true if the circle is not a great circle; if it is a great circle it belongs with at least a half great circle to the half sphere $H S^{n}\left(p_{0}\right)$ on which the stretching of a tangent vector is bounded by $1 / \sqrt{\kappa}<2$.

We consider now the induced map $h_{\Gamma}: \Gamma\left(S^{n}\right) \rightarrow \Pi(M)$. We can modify $h_{r}$ 
in such a manner that the parameterization on the image circles is proportional to the arc length. By keeping the same notations, we therefore can assume $h_{r}\left(\Gamma\left(S^{n}\right)\right) \subset \Pi^{c^{*}-}(M)$ with $c^{*}=8 \pi^{2}$. From $\S 1$ we then have the existence of $g(n)$ different closed geodesics of positive $E$-value $<8 \pi^{2}$, i.e., of length $<4 \pi$. But such geodesics must be simple and of length $\geq 2 \pi$.

Assume now that $\kappa \sim 0.46$ is the solution of the equation $2 \sqrt{\kappa} \sin \frac{\pi}{2 \sqrt{\kappa}}$ $=1$. Consider a circle $b$ on $H S^{n}\left(p_{1}\right)$ parallel to the equator $E S^{n}\left(p_{0}, p_{1}\right)$ and having distance $\sin r$ from $p_{1}$, measured on $S^{n}$. Such a circle has length $2 \pi$ $\sin r$. From (ii) it follows that the length of the image of $b$ under $h$ is bounded by $2 \pi \sin (r / \sqrt{\kappa}) /\left(\sqrt{\kappa} \sin \frac{\pi}{2 \sqrt{\kappa}}\right) \leq 4 \pi$; actually the length is even $<4 \pi$. This is clear for $r<\pi / 2$; for $r=\pi / 2$, the circle $b$ lies on $H S^{n}\left(p_{0}\right)$ and then we can apply (i).

If we denote by $\Gamma\left(S^{n-1}\right)$ the subspace of $\Gamma\left(S^{n}\right)$ formed by the circles parallel to $E S^{n}\left(p_{0}, p_{1}\right)$ we can assume that $h_{\Gamma}\left(\Gamma\left(S^{n-1}\right)\right) \subset \Pi^{c^{*}-}(M)$ with $c^{*}=8 \pi^{2}$. Thus we have the existence of $g(n-1)$ simple closed geodesics on $M$ with length in $[2 \pi, 4 \pi[$.

Assume finally that the non-trivial geodesics on $M$ with length in [ $2 \pi, 4 \pi[$ are not isolated or are non-degenerate. In the first case there then are infinitely many simple closed geodesics with length in $[2 \pi, 4 \pi[$. In the second case we deduce from the Morse theory that two linearly independent homology classes $z_{1}$ and $z_{2}$ of $\Pi(M) \bmod \Pi^{\circ}(M)$ having the property that the critical points (=closed geodesics) $g\left(z_{1}\right)$ and $g\left(z_{2}\right)$ of $E$-values $c\left(z_{1}\right)$ and $c\left(z_{2}\right)$, respectively, at which these homology classes remain hanging (cf. $\S 1$ ), are prime can be assumed to be different. Since $\operatorname{dim} H_{*}\left(\Gamma\left(S^{n}\right), \Gamma^{0}\left(S^{n}\right)\right)=n(n+1) / 2$, under our assumption and with $\kappa \sim 0.64$ there are $n(n+1) / 2$ simple closed geodesics on $M$ with length in [ $2 \pi, 4 \pi$ [. Similarly, we get $(n-1) n / 2$ such geodesics if we assume $\kappa \sim 0.46$.

\section{References}

[1] M. Berger, Les variétés riemanniennes (1/4)-pincées, Ann. Scuola Norm. Sup. Pisa (3) 14 (1960) $161-170$.

[2] D. Gromoll, W. Klingenberg \& W. Meyer, Riemannsche Geometric im Grossen, Lecture notes in Mathematics, Vol. 55, Springer, Heidelberg, 1968.

[3] W. Klingenberg, Uber riemannsche Mannigfaltigkeiten mit positiver Krümmung, Comment. Math. Helv. 35 (1961) 47-54.

[4] - Closed geodesics, to appear in Ann. of Math. Cf. also D. Craemer \& P. Flaschel, Geschlossene Geodätische, Lecture notes, Institute of Mathematics, University of Bonn, in preparation.

[5] H. E. Rauch, A contribution to differential geometry in the large, Ann. of Math. 54 (1951) 38-55. 